$2013 / 8$

\title{
Think BETA - Systems for Participation in Urban Development
}

\author{
Helmut Eirund, University of Applied Sciences Bremen, Martin Koplin, M2C Institute of Applied Media Technology and \\ Culture, Carl Skelton, Gotham Innovation Greenhouse, Thorsten Teschke, University of Applied Sciences Bremen
}

\begin{abstract}
In the Think BETA think tank (funded by the German BMBF) new technologies for urban planning and participation are developed and tested. In our actual implementation of Betaville we focus on two different methodologies. Both systems foster visualization and collaboration. One system works with augmented reality (AR) technology plus collaboration at a multiple-touch-table or tablet, the other with a virtual reality (VR) technology.
\end{abstract}

\section{Keywords - Digital media, participation, urban development.}

"Think BETA Evolution of Smart Cities" is an international think tank funded by the German Federal Ministry of Education with RTU as a partner. Think Beta is about research and development (R\&D) and the design of future technical and cultural infrastructure for the urban development of Smart Cities and the optimization of municipal services, participation infrastructure and environments for intercultural and diversity management optimization. The technical objective is to research and develop a mobile-stationary, multimedia environment as infrastructure for smart cities and their participative development [6]. Challenges are the requirements of a future technical and cultural (mass player) infrastructure. Existing technical approaches from eGovernance, urban planning, LBS, to the user-affected eCulture, eCreativity are integrated to develop and provide optimized mobilestationary digital systems for urban development, planning and participation. Previous software approaches did not take into account existing expertise, e.g., in the sectors of civic participation: integration of a decentralized design or the social balance in architecture was not adequately developed or focused on a single discipline perspective. This is to be counteracted through the interdisciplinary configuration of the think-tank and its experimental approach via urban art and design. Similarly, technical and organizational issues of participatory urban planning with different approaches for different user groups are considered in the actual systems of Betaville (an open-source multiplayer environment for real cities) and concepts for future ICT systems [5].

Current implementation of the Betaville idea provides two different methodologies. While both systems foster visualization and collaboration in urban planning, they use different technologies. One system works with augmented reality (AR) technology plus collaboration at a multiple-touchtable, the other one with a virtual reality (VR) technology. The next two sections are dedicated to the first approach; Section IV focuses on the VR approach. The last section presents the project background of the "Think BETA" idea and concludes the paper.

\section{Application Scenario}

Betaville is an actual project for the development of a platform that fosters online collaboration and participation of community groups in urban development. It provides mobile$\mathrm{AR}$, multiple-touch-table and 3D-online tools and methods. It provides a development, communication and decision-making environment for local initiatives and project groups. Betaville supports the complete development process from the analysis of citizens' needs, specifying early-stage ideas and fostering discussions through ongoing engagement of communities in project development and consensus formation: In Alphaville, a fictitious city, an old factory has been torn down. The vacant area is to be revived in the near future and the city hall constitutes a planning board. The official process has started. In order to take into consideration its citizens' demands for a livable city on the one hand as well as potential interests of authorities and technical restrictions on the other, the public administration is interested in the active participation of other parties in the decision and development process. Therefore, it creates a new project within Betaville and configures the available real estate in the virtual system.

Bob likes to actively take part in the planning process about his vicinity. As he is interested in a mixed use of the area, he uses Betaville's functionality to incorporate 3D models of the settlement of town houses as well as a small shopping mall with space for different shops. Alice gets to see Bob's proposal on Betaville and adds a 3D bounding box that serves as a request for a kindergarten that she finds essential for a vivid quarter. Later, other members of the community may create new proposals and replace her placeholder with sophisticated 3D models of kindergartens. After releasing her ideas, her friend Carol also is willing to participate in the redevelopment of the area. Equipped with her mobile device, Carol inspects the area and uses Betaville's mobile client for 3D on-site-visualizations of the different planning proposals on her mobile screen. With these authentic impressions in mind she realizes the long distance from the housing area to the kindergarten and changes the proposal directly on her mobile by repositioning the kindergarten. Back at home she realizes a lack of green space and substitutes the shopping mall in Bob's design by a small park. In order to integrate as many citizens as possible in the process, Alphaville additionally allocates interactive urban screens in the vivid city centre for communicating the current status of the development process. At multi-touch tables (MTT), small groups can meet and collaborate in real life, discuss alternative proposals, create and manipulate new ideas and visualize them on the attached urban screen. 
II. The Betaville Augmented Reality Approach: Urban Development on Site: The Mobile Client

While Betaville's web client is dedicated to the management of urban development projects and the multi-touch table fosters collaborative work on development projects, the mobile client leverages its mobility and location-awareness features: using the camera, GPS and compass that are built-in in the current generation of mobile phones, it provides the user with impressions of development proposals right on the spot.

The Betaville mobile client initially offers to find development projects either in a list view or a map view (Figure 1). The map view presents a map that is centred at the user's current location and shows nearby projects. As usual, the map can be zoomed and scrolled to look for projects in a wider area. Each project is depicted using an outline of the project area and "if clicked on" a flag shows summary information. The list view offers additional search features. In addition to a list of nearby projects, the user may search for a list of projects ordered by last edit date or by entering a search term.

If the user selects a project in either a map view or a list view, a project overview screen is shown with information on the project and further navigation options close to the top of the screen. In a properly set-up project, the info tab will provide textual information on the project intentions, purpose, or framing conditions along with a thumbnail map view of the project bounds, as well as activity information. The discussions tab gives space to discussions among active users, thus, fostering the exchange and cooperation among otherwise individual users. The proposals tab initially shows a list of root proposals, i.e., alternative urban development plans suggested by users.

As soon as the user selects one proposal, descriptive information on the proposal is shown similar to the project overview. Due to the hierarchical organization of proposals within the Betaville system, the user is now able to drill down into sub-proposals (if existing), i.e., proposals that are based on the currently presented proposal and that suggest changes such as the exchange of a building for a park or the re-positioning of a house.

If a proposal seems interesting and the user is nearby the project location, the AR [1] mode can be activated, which shows a prospectively correct view of the real location overlaid with the proposed 3D models. Here, the mobile client shows its true potential: the user may walk around the project area and watch the virtual buildings contained in the proposal from each desired perspective. The impressions collected by the user in this situation may stimulate further user activities, such as engaging in discussions, voting for or against a proposal or designing a sub-proposal that improves the proposal according to the user's on-site experience. One commonly known problem comes with the unprecise localisation through GPS and sensors by the mobile phone. Small errors in the localisation of the camera position leads to wrong perspectives of the 3D models. We currently work on markerless tracking systems that utilize pattern matching algorithms, similar to commercially available systems [8].

If the user finds a perspective particularly valuable, she may create the so-called freezes in the AR mode. A freeze is nothing more than a photo of the current view with attached information on the photograph's position and orientation. When uploaded to

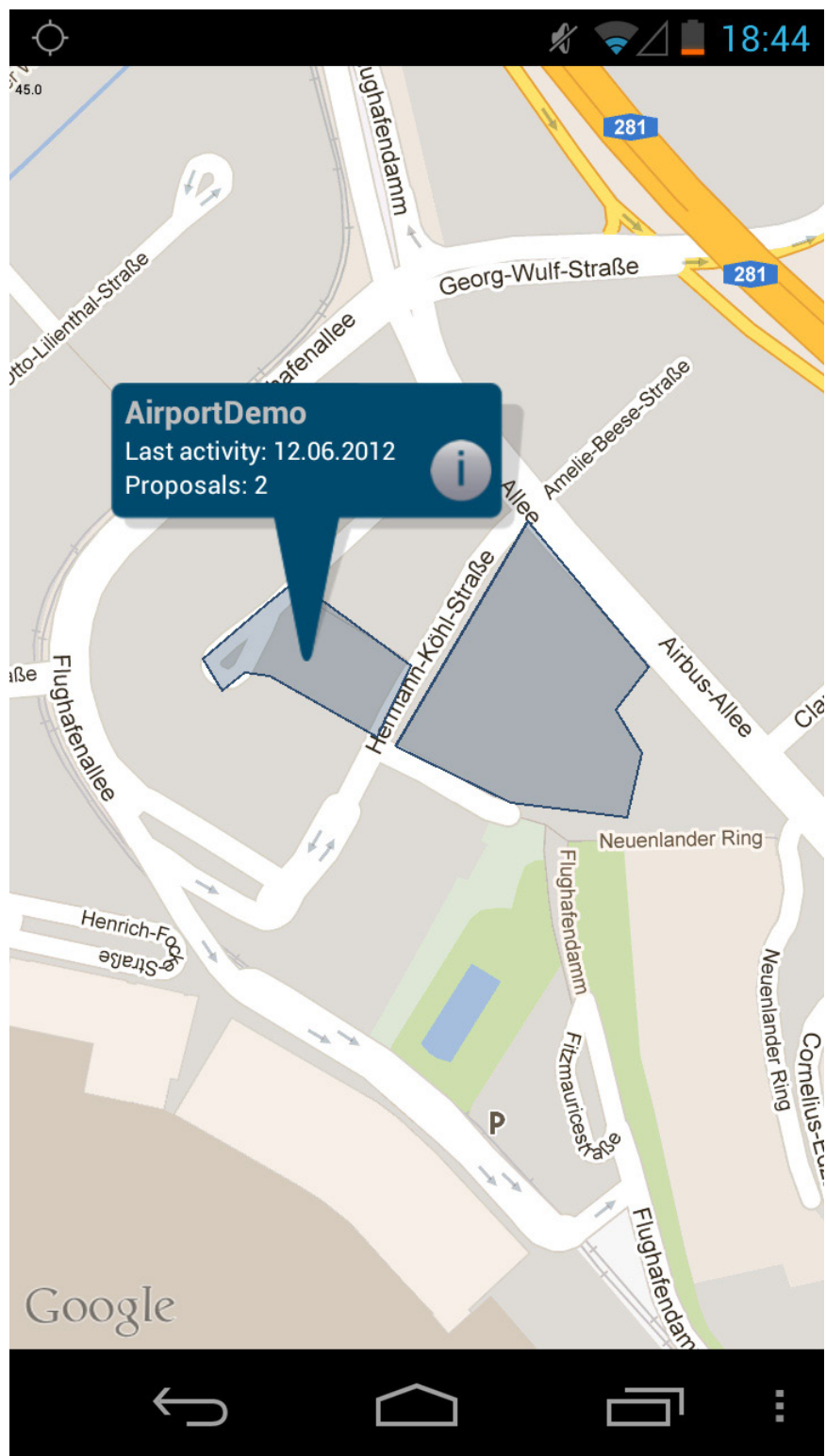

Fig. 1. Development projects in mobile map view.

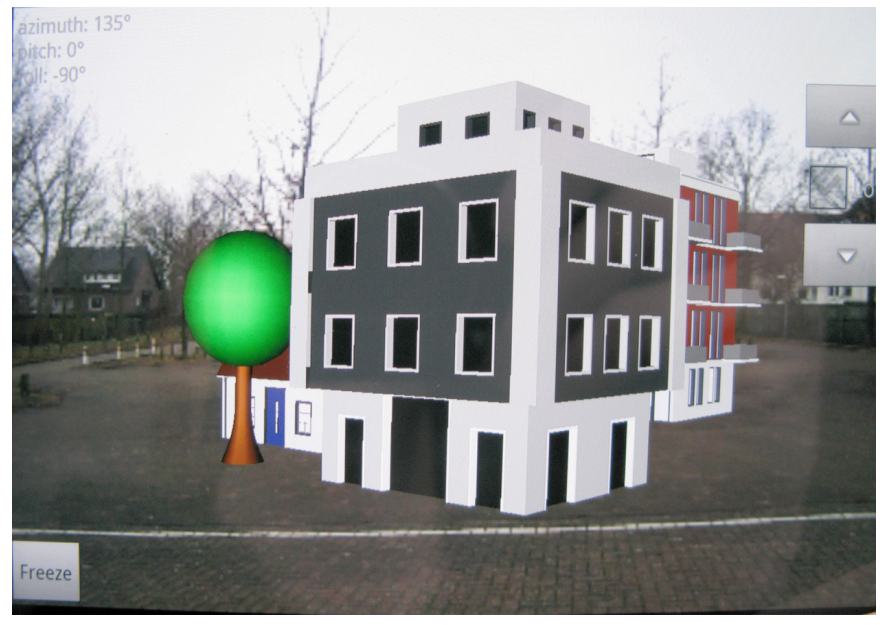

Fig. 2. Augmented reality view in the mobile client. 


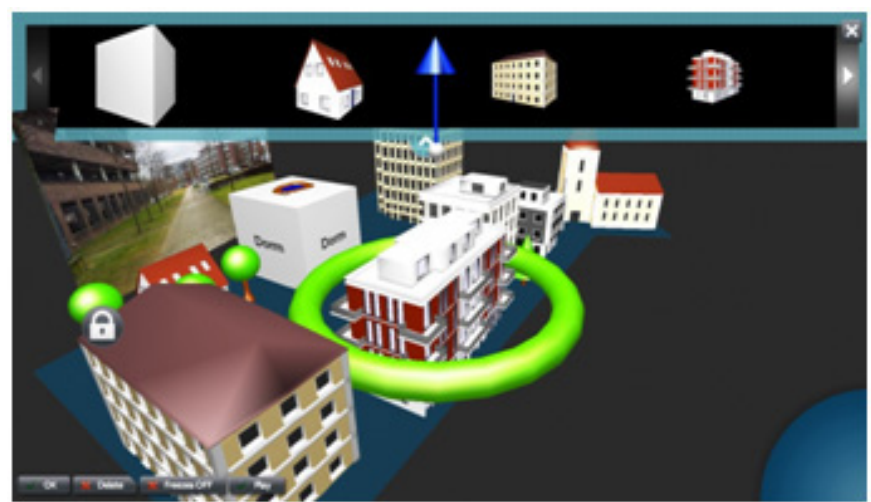

Fig. 3. Creation view on the multi-touch table to create and manipulate 3D proposals.

the Betaville server, these freezes allow other users to perceive an AR like situation everywhere: just like in the AR mode, a proposal can be overlaid prospectively correct on the freeze, thus making the invaluable combination of the real project area with the virtual 3D proposal available to users of the multi-touch table or mobile users who cannot be on the site.

MTTs offer a large screen, where users come together and interact with the system at the same time in an intuitive way. This technology offers, therefore, the possibility to enhance the faceto-face collaboration of small user groups [3]. The users are able to browse the hierarchical version tree of uploaded proposals and create a new version of an existing proposal by accessing the creation view. In the creation view, the $3 \mathrm{D}$ models of the parent proposal are presented initially. In addition, freezes are placed in this $3 \mathrm{D}$ environment with respect to the GPS coordinates and orientation of the photographer. This AR feature provides the users with impressions of the proposal embedding into its real environment without the need to leave the table.

Whenever a user taps on a 3D model its manipulation mode is activated, which places a ring around the model and an arrow above it. These tools enable the user to translate, scale, delete, and rotate the respective 3D model. When a user drags the uparrow, the model's altitude is changed. When he drags the ring that is placed around the model, the model is rotated (Figure 3).

\section{The Betaville Virtual Reality Approach: Urban DeVElopment IN THE WeB}

The Betaville Web platform combines David Gelernter's [4] concept of online "Mirror Worlds" as information-rich navigable 3D models of cities with the Massively Multiplayer Online Game entertainment genre, 3D Geographic Information Survey (GIS) mapping, and distributed/collaborative software suites for architectural and urban design.

Since the inception of the technical development of the Betaville platform in 2008, the New York Betaville team has been able to build out and deploy the Betaville web platform with success in Brooklyn (the Downtown Brooklyn Commons project, 2011), Queens (Tomorrow 2.0/ReGenerations, in collaboration with the Queens Hall of Science, Louis Armstrong MS 227, and the Urban Assembly Gateway School), and Manhattan (Columbia University School of Architecture and Planning). More deployments are underway in Toronto, Istanbul,
New York, and Busan. Betaville has moved from NYU Polytechnic, along with its project leaders, to the newly established Gotham Innovation Greenhouse. The technical platform is now robust, and readily implementable by third parties. To be effective as a public medium of discussion and concept development, the Betaville MMO platform has several specific design characteristics:

It will run on consumer-level hardware, and can be set up with basic-to-intermediate Java and PHP expertise. The ability to found a Betaville or to secede from one if the first is not congenial is, therefore, always available. No one faction or proponent can own a Betaville.

The visualization and interaction schemes are closely based on a common "vernacular" common to professionals and citizens: interactive perspective renderings are equally intelligible to planners, designers, artists, and the general public, providing for the broadest level of understanding; augmentation of this through the use of the model's components as links to live metadata and/ or external information provides for an environment to support informed participation, including contribution to the background information resources as one of the modes of contribution.

The Betaville web platform provides for the review and contribution of multiple versions of multiple concepts in parallel, at any level of detail. This makes it practical to engage broad participation early in the concept development process, when it is most useful and least expensive to follow through with.

The Betaville web platform conforms to open GIS and web standards to be able to aggregate other open data resources as particular use cases may require over time, from OpenStreetMaps to pertinent open government databases.

As a FreeBSD resource, the platform itself is open and available to participatory development, and is in any case available for detailed technical examination and/or adaptive re-use and extensions. The Betaville web platform can accept proposals for new concepts in the form of a combination of a .dae 3D model in the online world in any number of variations; the Collada file format it accepts can be exported from any level of authoring software, from the free Trimble SketchUp and Blender3D applications to professional design tools like Rhino and Revit. This provides for an easy learning curve and/ or minimal adaptation of modelling workflows for all levels of expertise. The Betaville web client, server, and accessory web services are available online under a FreeBSD license.

\section{BetaVille in Think BETA}

Smart Cities - Smart Culture - Smart Humans: The goal of the think tank is to network and promote trend-setting innovative art, research and development of technologies, methodologies and new services. Think Beta is about the design of future technical and cultural infrastructure for the urban development of Smart Cities and the optimization of municipal services and infrastructure or environments for intercultural and diversity management optimization. Media art in urban development, geo-economical activities, smart energy networks and ICT infrastructure for decentralized urban management also contain the Think BETA working areas. In 2011, the interdisciplinary knowledge exchange, cooperative research and art project design, the exchange of researchers and artists were in the foreground. In 
2012, more research projects were established and implemented. In 2013/14 a virtual living lab as a European Virtual Think BETA Research Institute will be established by its active members. The BxmC of New York University will establish a North-American Think BETA Design Lab that will be the partner organization of the European Virtual Think BETA Research Institute.

The first project Betaville started even before the Think BETA began its work. The Betaville project was launched as a joint initiative of the Brooklyn Experimental Media Centre (BxmC) of Polytechnic Institute of New York University and the M2C Institute for Applied Media Technology and Culture at the University of Applied Sciences of Bremen in July 2008, at a symposium for International Urban Media hosted by the M2C Institute in Bremen. Betaville has matured from a software art experiment into a vehicle of creative collaboration and exchange: a tool not only for planning and architecture studios, but a fully public medium for concept development and advocacy in the context of local art and urbanism projects. Betaville is a tool to express the imaginable ideas about our cities, their design and their future. Betaville is the proverbial science fiction tool for the utilization and realization of new cultural expressions in context of the development of our urban environment. The Betaville project is flanked by the second project of Think BETA artMuse. It started in early 2011 through EU-EACEA funding. artMuse facing the future - from industrial to post digital revolution - is an European art \& science project. In the media art part, the aim is to join contemporary media art to industry culture and generate artistic ideas for future urban and social developments. It establishes ties between the industrial and the digital revolution, sites of industrial heritage from the past to the future. In the science part, it is about participation and integration in new collaborative creative processes. The results were presented at media festivals in public space in Bocholt, Bitola and Gdansk, Delmenhorst, Ghent and Sofia. These places are conjoined from the historical influences by the early European industrialization to the digital revolution and both effects on the urban development and art. From this point of change in urbanity, artMuse takes a chance to look onto the next possible steps of future urban live, participative urban planning, urban design, and digital art. The third Think BETA R\&D project "TRAILS - CCBM - Crosscultural Business and Virtual Management Communication Analysing and Awareness Tool" refers directly to the human factor in future urban environments [7]. If the Betaville models get real as a future city, new educational aspects and learning in network methodologies, virtual communication and design processes, as well as social and cultural diversity will come to the fore. TRAILS - CCBM analyses and explores the needs and creates methodologies for training and education.

\section{Conclusions}

Within Betaville, members of the community, local authorities, or even potential investors have the chance to refine and extend the development branches created by Bob, Alice, and Carol by changing existing proposals or creating new ones from scratch. Furthermore, every member of the community has the chance to participate in the decision-making process that will converge on a small set of favoured solutions. Currently, Betaville experiments with different strategies to perform this task. Nevertheless, participation in the urban environment covers many categories of interest: city (re-)development, urban art, preserving cultural heritage, urban resting places, etc. Within the partners of Think BETA several project ideas in these fields have already been carried out successfully. The question is how to come to a generalized framework to generate participation in the new urban project design. At the same time, this will be discussed at the 53rd International Scientific Conference of Riga Technical University, the BMW Guggenheim Lab Berlin, the Municipal Art Society of New York City and the Goethe Institute Montreal. We invite the conference participants at RTU to take part in the discussion and become a partner in our future interdisciplinary projects. At present, we have finalized the AR and VR prototype systems. Demonstration videos are accessible through [2].

\section{REFERENCES}

1. Sa, M. de, Churchill, E. F., Isbister, K. Mobile Augmented Reality : Design Issues and Opportunities. Proceedings of the 13th International Conference on Human Computer Interaction with Mobile Devices and Services, MobileHCI 2011, Stockholm, Sweden, August 30 - September 2, 2011.p. 749-752. doi:10.1145/2037373.2037504

2. Betaville videos [online]. Betaville [cited 12.12.2012.]. http://betaville.hsbremen.de/techs.php, http://bxmc.poly.edu/betaville,

3. Clifton, P., Mazalek, A., Sanford, J., Rébola, C., Lee, S., Powell, N. SketchTop: Design Collaboration on a Multi-touch Tabletop. Proceedings of the 5th International Conference on Tangible, Embedded and Embodied Interaction (TEI'11), ACM, New York, NY, p. 333-336.

4. Gelernter, D. Mirror Worlds: Or, the Day Software Puts the World in a Shoebox... How It Will Happen, and What It Will Mean. New York: Oxford, 1992, $256 \mathrm{p}$.

5. What is Betaville? [online]. Betaville.net [cited 12.12.2012.]. http://betaville.net

6. Benouaret, K., Valliyur-Ramalingam, R., Charoy, F. CrowdSC: Building Smart Cities with Large Scale Citizen Participation. IEEE Internet Computing. Vol. 17, No. 6, 2003, p. 57-63. doi:10.1109/MIC.2013.88

7. Sancristobal, E., Martin, S., Gil, R., Orduna, P., Tawfik, M., Pesquera, A., Diaz, G., Colmenar, A., Garcia-Zubia, J., Castro, M. State of Art, Initiatives and New Challenges for Virtual and Remote Labs. IEEE 12th International Conference on Advanced Learning Technologies, July 4-6, 2012. p. 714-715.

8. Location-based AR [online]. Metaio Developer Portal [cited 18.12.2013.]. http://dev.metaio.com/sdk/tutorials/location-based-ar 


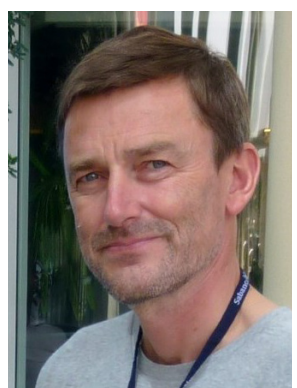

Helmut Eirund, Diploma in Computer Science (1985, University Kiel, Germany), Researcher at TA Olivetti Research Lab (Nurnberg), Project Leader in CEC funded projects on multimedia documents, Ph.D. thesis on conceptual structures in multimedia documents (1991, University Oldenburg). In 1994, Professor of Media Informatics at the University of Applied Sciences, Harz (Germany); since 2001 Professor of Media Informatics at the University of Applied Sciences, Bremen (Germany). He was a Visiting Professor at California State University in Arcata 2005. He is a Co-founder and Scientific Director of M2C, Bremen. Research interests: mobile computing, electronic entertainment, interaction design.

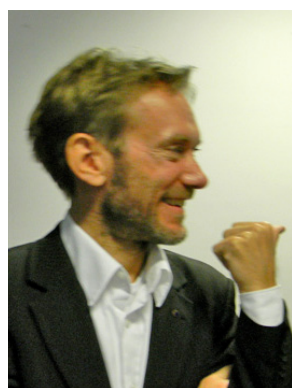

Martin Koplin, one of the founders and Director of the M2C Institute for Applied Media Technology and Culture at the University of Applied Sciences, Bremen, Germany. He has worked as a Media Artist for over 15 years and as a Scientific Researcher in the field of digital media and digital culture for more than 10 years. He studied digital media communication, cultural studies, organizational studies and fine arts. $\mathrm{He}$ holds two master's degrees, university certificate in Neuroenergetic Leadership and Organisational Development and actually is completing his doctoral thesis in Computer Sciences. He is a Scientist in Residence at the BxmC of the Polytechnic Institute of New York University.

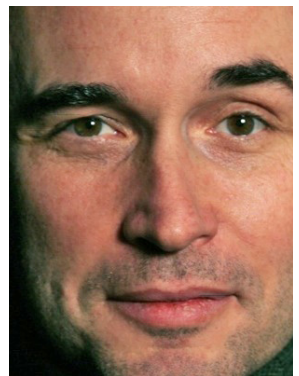

Carl Skelton, Director of the Gotham Innovation Greenhouse, and a Candidate for the Dr.Ing. in Informatics at the University of Bremen. His creative/ research work bridges the arts, design, technology, and community engagement. $\mathrm{He}$ is currently working on two books: New Soft City Culture: The Case of Betaville for Springer, and The Multimedia Programming Fakebook with R. Luke DuBois for MIT Press. Creative projects include Betaville, a massively participatory editable mirror world project with an international network of partners and collaborators. You can learn more at betaville.net. From 2003 to 2012, Carl founded and directed the Brooklyn Experimental Media Centre and the academic programs in Integrated Digital Media at the Polytechnic Institute of New York University. Carl's work has been supported by Microsoft Research, the Rockefeller Foundation through its Cultural Innovation Fund, the National Science Foundation, the Ontario Arts Council, and the Canada Council for the Arts.

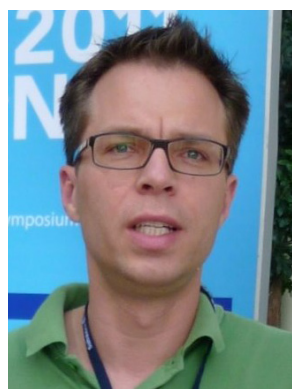

Thorsten Teschke, Diploma in Computer Science (1998, University Oldenburg, Germany), Visiting Scholar at Carnegie Mellon University (CMU) in Pittsburgh, PA, USA (February to July 1998), doctoral thesis on semantic questions in componentbased software development (2003, University Oldenburg, Germany). He has been a Professor of Software Engineering at the University of Applied Sciences, Bremen since 2005.

Research interests: mobile computing, context-aware systems, software architecture, web applications.

\section{CONTACT Data}

Helmut Eirun

University of Applied Sciences Bremen and M2C Institute Address: Flughafenallee 10, D-28199 Bremen, Germany

Phone: +49 42159055401

E-mail: helmut.eirund@hs-bremen.de

Martin Koplin

M2C Institute of Applied Media Technology and Culture Address: Flughafenallee 10, D-28199 Bremen, Germany Phone: +49 42159055402

E-mail: koplin@m2c-bremen.de

Carl Skelton

Gotham Innovation Greenhouse

111 West 57th Street, Suite 1600, New York, NY 10019, USA

Phone: +01 347-705-9900

E-mail: carl@gothaminnovationgreenhouse.com

Thorsten Teschke

University of Applied Sciences Bremen

Address: Flughafenallee 10, D-28199 Bremen, Germany

Phone: +49 42159055428

E-mail: thorsten.teschke@hs-bremen.de 